Vietnam Journal of Mechanics, VAST, Vol.33, No. 3 (2011), pp. $131-147$

\title{
NONLINEAR POSTBUCKLING OF ECCENTRICALLY STIFFENED FUNCTIONALLY GRADED PLATES AND SHALLOW SHELLS
}

\author{
Dao Huy Bich ${ }^{1}$, Vu Hoai Nam ${ }^{2}$, Nguyen Thi Phuong ${ }^{2}$ \\ ${ }^{1}$ Hanoi University of Science, $V N U$ \\ ${ }^{2}$ University of Transport Technology, Hanoi, Vietnam
}

\begin{abstract}
The paper deals with the formulation of governing equations of eccentrically stiffened functionally graded plates and shallow shells based uppon the classical shell theory and the smeared stiffeners technique taking into account geometrical nonlinearity in Von Karman-Donnell sense. Material properties are assumed to be temperatureindependent and graded in the thickness direction according to a simple power law distribution in terms of the volume fraction of constituents. The shells are reinforced by eccentrically longitudinal and transversal stiffeners made of full metal or full ceramic depending on situation of stiffeners at metal-rich side or ceramic-rich side of the shell respectively. Obtained governing equations can be used in research on nonlinear postbuckling of mentioned above structures. By use of the Galerkin method an approximated analytical solution to the nonlinear stability problem of reinforced FGM plates and shallow shells is performed. The postbuckling load-deflection curves of the shells are investigated and analytical expressions of the upper and lower buckling loads are presented. A comparison of the effectiveness of stiffeners in enhancing the stability of FGM plates and shallow shells is given.
\end{abstract}

Key words: Functionally graded material, plates, shallow shells, stiffeners, upper and lower buckling loads, nonlinear postbuckling.

\section{INTRODUCTION}

Functionally graded materials (FGMs) have properties varying smoothly through the thickness by changing continuously in the volume fractions of their constituents. Reinforced FGM structures like plates and shallow shells may be used in aerospace vehicles and nuclear reactors. They can be found in aircraft components as primary load carrying structures such as fuselage sections as well as in spacecraft and missile structural applications. The stiffening member provides the benefit of added load-carrying static and dynamic capability with a relatively small additional weight penalty. Thus study on static and dynamic problems of reinforced FGM plates and shallow shells with geometrical nonlinearity are of significant practical interest. 
In recent years, many important studies have been researched about stability and vibration of FGM plates and shells without stiffeners. In the buckling research field, Birman [1] presented a formulation of stability problem for FGM plates subjected to uniaxial compression; Feldman and Aboudi [2] studied elastic bifurcation of FGM plates acted on by compressive loading, Tung and Duc [3] investigated buckling and postbuckling of FGM plates under compressive and thermal loads. Nonlinear analysis on buckling and postbuckling of axially loaded and pressure-loaded FGM cylindrical panels in thermal environments has been found in works of Shen [4], Shen and Leung [5], Duc and Tung [6], Yang et al [7]. In the work [8] Li and Batra investigated buckling of axially compressed thin cylindrical shell with FGM middle layer, Shen [9] and Huaiwei Huang [10] presented research on nonlinear postbuckling of FGM cylindrical shells under radial loads by use of the nonlinear large deflection theory of cylindrical shell. Bich [11] studied nonlinear buckling analysis of FGM shallow spherical shells under external pressure by analytical approach and Naj et al. [12] investigated pressure-loaded FGM conical shells. In the field of dynamic buckling, Sofiyev [13], Ng et al. [14], Darabi et al. [15], Bich and Long [16] presented dynamic buckling analysis of FGM cylindrical shells under various loadings and Ganapathi [17] established analysis for FGM shallow spherical shells.

However, the nonlinear buckling analysis of reinforced functionally graded plates and shells has received comparatively little attention, this may be because of their inherent complexity. In this paper, the formulation of governing equations of eccentrically stiffened functionally graded plates and shallow shells based uppon the classical shell theory and smeared stiffeners technique is represented taking into account geometrical nonlinearly in Von Karman-Donnell sense. The nonlinear response of stiffened FGM plates and shallow shells under various loading is investigated by an analytical approach. The resulting equations are solved by Garlekin method to obtain closed-form expressions of the upper and lower buckling loads and nonlinear post-buckling load-deflection curves. A comparison of the effectiveness of stiffeners in enhancing the stability of FGM plates and shallow shells is given.

\section{GOVERNING EQUATIONS}

\subsection{Functionally graded material (FGM)}

FGMs are microscopically inhomogeneous materials, in which material properties vary smoothly and continuously from one surface of the material to the other surface. These materials are made from a mixture of ceramic and metal, or a combination of different materials. A such mixture of ceramic and metal with a continuously varying volume fraction can be manufactured. Especially FGM thin-walled structures with metal in inner surface and ceramic in outer surface are widely used in practice. Assume that the modulus of elasticity $E$ changes in the thickness direction $z$, while the Poisson ratio $\nu$ is assumed to be constant. Denote $V_{m}$ and $V_{c}$ being volume - fractions of metal and ceramic phases respectively, which are related by $V_{m}+V_{c}=1$ and $V_{c}$ is expressed as $V_{c}(z)=$

$\left(\frac{2 z+h}{2 h}\right)^{k}$, where $h$ is the thickness of thin-walled structure, $k$ is the volume-fraction exponent $(k \geq 0)$. Then the elasticity modulus and the Poisson ratio of functionally 
graded material can be evaluated as following

$$
E(z)=E_{m} V_{m}+E_{c} V_{c}=E_{m}+\left(E_{c}-E_{m}\right)\left(\frac{2 z+h}{2 h}\right)^{k}, \quad \nu(z)=\nu=\text { const. }
$$

The values with subscripts $m$ and $c$ belong to metal and ceramic, respectively.

\subsection{Eccentrically stiffened functionally graded shallow shells}

Consider a doubly curved functionally graded shallow shell of thickness $h$ and in - plane edges $a$ and $b$. The shell reinforced by eccentrically longitudinal and transversal stiffeners is shown in Fig. 1. The shallow shell is assumed to have a relative small rise as compared with its span. Let the $\left(x_{1}, x_{2}\right)$ plane of the Cartesian coordinates overlaps the rectangular plane area of the shell. The middle surface of the shell generally is defined in terms of curvilinear coordinates, but for the shallow shell the Cartesian coordinates can replace the curvilinear coordinates on the middle surface.

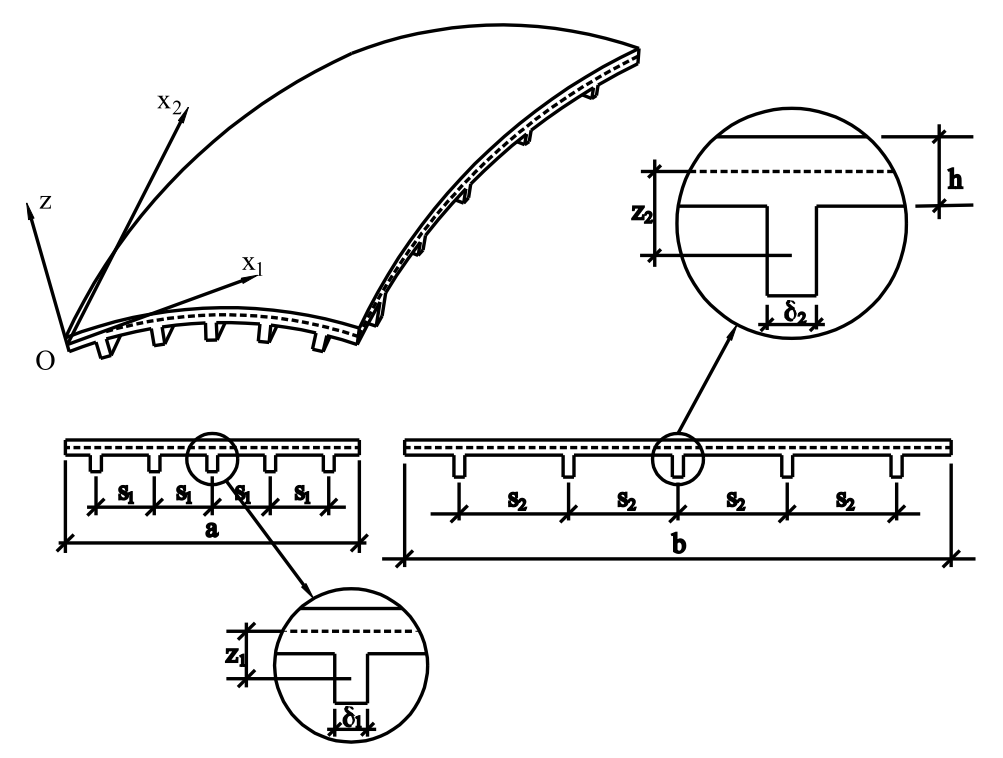

Fig. 1. Configuration of an eccentrically stiffened shallow shells

In the present study, the classical shell theory and the Lekhnitsky smeared stiffe ners technique are used to obtain the equilibrium and compatibility equations as well as expressions of buckling loads and nonlinear load-deflection curves of eccentrically stiffened FGM shallow shells.

The strains across the shell thickness at a distance $z$ from the mid-surface are

$$
\varepsilon_{1}=\varepsilon_{1}^{0}-z \chi_{1}, \quad \varepsilon_{2}=\varepsilon_{2}^{0}-z \chi_{2}, \quad \gamma_{12}=\gamma_{12}^{0}-2 z \chi_{12},
$$

where $\varepsilon_{1}^{0}$ and $\varepsilon_{2}^{0}$ are normal strains, $\gamma_{12}^{0}$ is the shear strain at the middle surface of the shell and $\chi_{i j}$ are the curvatures.

According to the classical shell theory the strains at the middle surface and curvatures are related to the displacement components $u, v, w$ in the $x_{1}, x_{2}, z$ coordinate 
directions as [18].

$$
\begin{array}{ll}
\varepsilon_{1}^{0}=\frac{\partial u}{\partial x_{1}}-k_{1} w+\frac{1}{2}\left(\frac{\partial w}{\partial x_{1}}\right)^{2}, & \chi_{1}=\frac{\partial^{2} w}{\partial x_{1}^{2}}, \\
\varepsilon_{2}^{0}=\frac{\partial v}{\partial x_{2}}-k_{2} w+\frac{1}{2}\left(\frac{\partial w}{\partial x_{2}}\right)^{2}, & \chi_{2}=\frac{\partial^{2} w}{\partial x_{2}^{2}} \\
\gamma_{12}^{0}=\frac{\partial u}{\partial x_{2}}+\frac{\partial v}{\partial x_{1}}+\frac{\partial w}{\partial x_{1}} \frac{\partial w}{\partial x_{2}}, & \chi_{12}=\frac{\partial^{2} w}{\partial x_{1} \partial x_{2}},
\end{array}
$$

where geometrical nonlinearity in von Karman-Donnell sense is accounted and $k_{1}=\frac{1}{R_{1}}, k_{2}=$ $\frac{1}{R_{2}}$ are principal curvatures of the shell, $R_{1}, R_{2}$ are radii of curvatures.

From Eqs.(2) the strains must be relative in the deformation compatibility equation

$$
\frac{\partial^{2} \varepsilon_{1}^{0}}{\partial x_{2}^{2}}+\frac{\partial^{2} \varepsilon_{2}^{0}}{\partial x_{1}^{2}}-\frac{\partial^{2} \gamma_{12}^{0}}{\partial x_{1} \partial x_{2}}=\left(\frac{\partial^{2} w}{\partial x_{1} \partial x_{2}}\right)^{2}-\frac{\partial^{2} w}{\partial x_{1}^{2}} \frac{\partial^{2} w}{\partial x_{2}^{2}}-k_{1} \frac{\partial^{2} w}{\partial x_{2}^{2}}-k_{2} \frac{\partial^{2} w}{\partial x_{1}^{2}} .
$$

The constitutive stress-strain equations by Hooke law for the shell material are omitted here for brevity. The contribution of stiffeners can be accounted for using the Lekhnitsky smeared stiffeners technique. Then integrating the stress-strain equations and their moments through the thickness of the shell, the expressions for force and moment resultants of an eccentrically stiffened FGM shallow shell are obtained.

$$
\begin{aligned}
& N_{1}=\left(A_{11}+\frac{E A_{1}}{s_{1}}\right) \varepsilon_{1}^{0}+A_{12} \varepsilon_{2}^{0}-\left(B_{11}+C_{1}\right) \chi_{1}-B_{12} \chi_{2}, \\
& N_{2}=A_{12} \varepsilon_{1}^{0}+\left(A_{22}+\frac{E A_{2}}{s_{2}}\right) \varepsilon_{2}^{0}-B_{12} \chi_{1}-\left(B_{22}+C_{2}\right) \chi_{2}, \\
& N_{12}=A_{66} \gamma_{12}^{0}-2 B_{66} \chi_{12}, \\
& M_{1}=\left(B_{11}+C_{1}\right) \varepsilon_{1}^{0}+B_{12} \varepsilon_{2}^{0}-\left(D_{11}+\frac{E I_{1}}{s_{1}}\right) \chi_{1}-D_{12} \chi_{2}, \\
& M_{2}=B_{12} \varepsilon_{1}^{0}+\left(B_{22}+C_{2}\right) \varepsilon_{2}^{0}-D_{12} \chi_{1}-\left(D_{22}+\frac{E I_{2}}{s_{2}}\right) \chi_{2}, \\
& M_{12}=B_{66} \gamma_{12}^{0}-2 D_{66} \chi_{12},
\end{aligned}
$$

where $A_{i j}, B_{i j}, D_{i j},(i, j=1,2,6)$ are extensional, coupling and bending stiffenesses of the shell without stiffeners.

$$
\begin{array}{lll}
A_{11}=A_{22}=\frac{E_{1}}{1-\nu^{2}}, & A_{12}=\frac{E_{1} \nu}{1-\nu^{2}}, & A_{66}=\frac{E_{1}}{2(1+\nu)}, \\
B_{11}=B_{22}=\frac{E_{2}}{1-\nu^{2}}, & B_{12}=\frac{E_{2} \nu}{1-\nu^{2}}, & B_{66}=\frac{E_{2}}{2(1+\nu)}, \\
D_{11}=D_{22}=\frac{E_{3}}{1-\nu^{2}}, & D_{12}=\frac{E_{3} \nu}{1-\nu^{2}}, & D_{66}=\frac{E_{3}}{2(1+\nu)},
\end{array}
$$


with

$$
\begin{aligned}
& E_{1}=\left(E_{m}+\frac{E_{c}-E_{m}}{k+1}\right) h, \quad E_{2}=\frac{\left(E_{c}-E_{m}\right) k h^{2}}{2(k+1)(k+2)}, \\
& E_{3}=\left[\frac{E_{m}}{12}+\left(E_{c}-E_{m}\right)\left(\frac{1}{k+3}-\frac{1}{k+2}+\frac{1}{4 k+4}\right)\right] h^{3},
\end{aligned}
$$

and

$$
C_{1}=\frac{E A_{1} z_{1}}{s_{1}}, \quad C_{2}=\frac{E A_{2} z_{2}}{s_{2}} .
$$

In above relations (4), (5) and (7) $E$ is the elasticity modulus in the axial direction of the corresponding stiffener which is assumed identical for both types of stiffeners. The spacings of the longitudinal and transversal stiffeners are denoted by $s_{1}$ and $s_{2}$ respectively. The quantities $A_{1}, A_{2}$ are the cross section areas of the stiffeners and $I_{1}, I_{2}, z_{1}, z_{2}$ are the second moments of cross section areas and eccentricities of the stiffeners with respect to the middle surface of the shell respectively.

Important remark. In order to provide continuity between the shell and stiffeners, thus stiffeners are made of full metal if putting them at the metal-rich side of the shell and conversely full ceramic stiffeners at the ceramic-rich side of the shell, consequently $E=E_{m}$ for full metal stiffeners and $E=E_{c}$ for full ceramic ones.

For using later, the reverse relations are obtained from Eqs. (4)

$$
\begin{aligned}
& \varepsilon_{1}^{0}=A_{22}^{*} N_{1}-A_{12}^{*} N_{2}+B_{11}^{*} \chi_{1}+B_{12}^{*} \chi_{2}, \\
& \varepsilon_{2}^{0}=A_{11}^{*} N_{2}-A_{12}^{*} N_{1}+B_{21}^{*} \chi_{1}+B_{22}^{*} \chi_{2}, \\
& \gamma_{12}^{0}=A_{66}^{*}+2 B_{66}^{*} \chi_{12},
\end{aligned}
$$

where

$$
\begin{aligned}
& A_{11}^{*}=\frac{1}{\Delta}\left(A_{11}+\frac{E A_{1}}{s_{1}}\right), A_{22}^{*}=\frac{1}{\Delta}\left(A_{22}+\frac{E A_{2}}{s_{2}}\right), A_{12}^{*}=\frac{A_{12}}{\Delta}, A_{66}^{*}=\frac{1}{A_{66}}, \\
& \Delta=\left(A_{11}+\frac{E A_{1}}{s_{1}}\right)\left(A_{22}+\frac{E A_{2}}{s_{2}}\right)-A_{12}^{2}, \\
& B_{11}^{*}=A_{22}^{*}\left(B_{11}+C_{1}\right)-A_{12}^{*} B_{12}, B_{22}^{*}=A_{11}^{*}\left(B_{22}+C_{2}\right)-A_{12}^{*} B_{12}, \\
& B_{12}^{*}=A_{22}^{*} B_{12}-A_{12}^{*}\left(B_{22}+C_{2}\right), B_{21}^{*}=A_{11}^{*} B_{12}-A_{12}^{*}\left(B_{11}+C_{1}\right), B_{66}^{*}=\frac{B_{66}}{A_{66}} .
\end{aligned}
$$

Substituting Eqs. (8) into Eqs.(5) yields

$$
\begin{aligned}
& M_{1}=B_{11}^{*} N_{1}+B_{21}^{*} N_{2}-D_{11}^{*} \chi_{1}-D_{12}^{*} \chi_{2}, \\
& M_{2}=B_{12}^{*} N_{1}+B_{22}^{*} N_{2}-D_{21}^{*} \chi_{1}-D_{22}^{*} \chi_{2}, \\
& M_{12}=B_{66}^{*} N_{12}-2 D_{66}^{*} \chi_{12},
\end{aligned}
$$


where

$$
\begin{aligned}
& D_{11}^{*}=D_{11}+\frac{E I_{1}}{s_{1}}-\left(B_{11}+C_{1}\right) B_{11}^{*}-B_{12} B_{21}^{*}, \\
& D_{22}^{*}=D_{22}+\frac{E I_{2}}{s_{2}}-B_{12} B_{12}^{*}-\left(B_{22}+C_{2}\right) B_{22}^{*}, \\
& D_{12}^{*}=D_{12}-\left(B_{11}+C_{1}\right) B_{12}^{*}-B_{12} B_{22}^{*}, \\
& D_{21}^{*}=D_{12}-B_{12} B_{11}^{*}-\left(B_{22}+C_{2}\right) B_{21}^{*}, \\
& D_{66}^{*}=D_{66}-B_{66} B_{66}^{*} .
\end{aligned}
$$

The nonlinear equilibrium equations of a shallow shell based on the classical shell theory are given by

$$
\begin{aligned}
\frac{\partial N_{1}}{\partial x_{1}}+\frac{\partial N_{12}}{\partial x_{2}}=0, \quad \frac{\partial N_{12}}{\partial x_{1}} & +\frac{\partial N_{2}}{\partial x_{2}}=0 \\
\frac{\partial^{2} M_{1}}{\partial x_{1}^{2}}+2 \frac{\partial^{2} M_{12}}{\partial x_{1} \partial x_{2}}+\frac{\partial^{2} M_{2}}{\partial x_{2}^{2}} & +N_{1} \frac{\partial^{2} w}{\partial x_{1}^{2}}+2 N_{12} \frac{\partial^{2} w}{\partial x_{1} \partial x_{2}}+ \\
& +N_{2} \frac{\partial^{2} w}{\partial x_{2}^{2}}+k_{1} N_{1}+k_{2} N_{2}+q_{0}=0 .
\end{aligned}
$$

Considering the first two of Eqs. (10), a stress function may be defined as

$$
N_{1}=\frac{\partial^{2} \phi}{\partial x_{2}^{2}}, \quad N_{2}=\frac{\partial^{2} \phi}{\partial x_{1}^{2}}, \quad N_{12}=-\frac{\partial^{2} \phi}{\partial x_{1} \partial x_{2}} .
$$

The substitution of Eqs. (8) into the compatibility Eqs. (3) and Eqs. (9) into the third of Eqs. (10), taking into account expressions (2) and (11), yields a system of equations

$$
\begin{aligned}
& A_{11}^{*} \frac{\partial^{4} \phi}{\partial x_{1}^{4}}+\left(A_{66}^{*}-2 A_{12}^{*}\right) \frac{\partial^{4} \phi}{\partial x_{1}^{2} \partial x_{2}^{2}}+A_{22}^{*} \frac{\partial^{4} \phi}{\partial x_{2}^{4}}+B_{21}^{*} \frac{\partial^{4} w}{\partial x_{1}^{4}}+\left(B_{11}^{*}+B_{22}^{*}-\right. \\
& \left.-2 B_{66}^{*}\right) \frac{\partial^{4} w}{\partial x_{1}^{2} \partial x_{2}^{2}}+B_{12}^{*} \frac{\partial^{4} w}{\partial x_{2}^{4}}+k_{1} \frac{\partial^{2} w}{\partial x_{2}^{2}}+k_{2} \frac{\partial^{2} w}{\partial x_{1}^{2}}=\left(\frac{\partial^{2} w}{\partial x_{1} \partial x_{2}}\right)^{2}-\frac{\partial^{2} w}{\partial x_{1}^{2}} \frac{\partial^{2} w}{\partial x_{2}^{2}}, \\
& D_{11}^{*} \frac{\partial^{4} w}{\partial x_{1}^{4}}+\left(D_{12}^{*}+D_{21}^{*}+4 D_{66}^{*}\right) \frac{\partial^{4} w}{\partial x_{1}^{2} \partial x_{2}^{2}}+ \\
& +D_{22}^{*} \frac{\partial^{4} w}{\partial x_{2}^{4}}-B_{21}^{*} \frac{\partial^{4} \phi}{\partial x_{1}^{4}}-\left(B_{11}^{*}+B_{22}^{*}-2 B_{66}^{*}\right) \frac{\partial^{4} \phi}{\partial x_{1}^{2} \partial x_{2}^{2}}- \\
& -B_{12}^{*} \frac{\partial^{4} \phi}{\partial x_{2}^{4}}-k_{1} \frac{\partial^{2} \phi}{\partial x_{2}^{2}}-k_{2} \frac{\partial^{2} \phi}{\partial x_{1}^{2}}-\frac{\partial^{2} \phi}{\partial x_{2}^{2}} \frac{\partial^{2} w}{\partial x_{1}^{2}}+2 \frac{\partial^{2} \phi}{\partial x_{1} \partial x_{2}} \frac{\partial^{2} w}{\partial x_{1} \partial x_{2}}-\frac{\partial^{2} \phi}{\partial x_{1}^{2}} \frac{\partial^{2} w}{\partial x_{2}^{2}}=q_{0} .
\end{aligned}
$$

Eqs. (12) and (13) are the basic equations used to investigate the stability of eccentrically stiffened functionally graded shallow shells. They are nonlinear equations in terms of two dependent unknowns $w$ and $\phi$.

For a FGM shallow shell without stiffeners by putting $A_{1}=A_{2}=0$ and $I_{1}=I_{2}=0$, Eqs. (12) and (13) automatically reduce to the ones given in [16]

$$
\frac{1}{E_{1}}\left(\frac{\partial^{4} \phi}{\partial x_{1}^{4}}+2 \frac{\partial^{4} \phi}{\partial x_{1}^{2} \partial x_{2}^{2}}+\frac{\partial^{4} \phi}{\partial x_{2}^{4}}\right)+k_{1} \frac{\partial^{2} w}{\partial x_{2}^{2}}+k_{2} \frac{\partial^{2} w}{\partial x_{1}^{2}}=\left(\frac{\partial^{2} w}{\partial x_{1} \partial x_{2}}\right)^{2}-\frac{\partial^{2} w}{\partial x_{1}^{2}} \frac{\partial^{2} w}{\partial x_{2}^{2}},
$$




$$
\begin{aligned}
\frac{E_{1} E_{3}-E_{2}^{2}}{E_{1}\left(1-\nu^{2}\right)}\left(\frac{\partial^{4} w}{\partial x_{1}^{4}}+2 \frac{\partial^{4} w}{\partial x_{1}^{2} \partial x_{2}^{2}}+\frac{\partial^{4} w}{\partial x_{2}^{4}}\right) & -k_{1} \frac{\partial^{2} \phi}{\partial x_{2}^{2}}-k_{2} \frac{\partial^{2} \phi}{\partial x_{1}^{2}}-\frac{\partial^{2} \phi}{\partial x_{2}^{2}} \frac{\partial^{2} w}{\partial x_{1}^{2}}+ \\
& +2 \frac{\partial^{2} \phi}{\partial x_{1} \partial x_{2}} \frac{\partial^{2} w}{\partial x_{1} \partial x_{2}}-\frac{\partial^{2} \phi}{\partial x_{1}^{2}} \frac{\partial^{2} w}{\partial x_{2}^{2}}=q_{0}
\end{aligned}
$$

\section{NONLINEAR BUCKLING ANALYSIS}

An approximation is acceptable in the vicinity of the buckling load. The FGM shell considered in the following analysis is simply supported and subjected to uniformly distributed pressure of intensity $q_{0}$ and lateral compressive loads of intensities $r_{0}$ and $p_{0}$ respectively at its cross section (in Pascals). Thus the boundary conditions considered in the current study are

$$
\begin{aligned}
& w=0, M_{1}=0, N_{1}=-r_{0} h, N_{12}=0, \text { at } x_{1}=0, x_{1}=a, \\
& w=0, M_{2}=0, N_{2}=-p_{0} h, N_{12}=0, \text { at } x_{2}=0, x_{2}=b .
\end{aligned}
$$

where $a$ and $b$ are the lengths of in-plane edges of the shallow shell.

The conditions (16) can be satisfied if the buckling mode shape is represented by

$$
w=f \sin \frac{m \pi x_{1}}{a} \sin \frac{n \pi x_{2}}{b}
$$

where $f$ is a maximum deflection, $m, n$ are odd natural numbers. Substituting (17) into Eqs.(12) and solving obtained equation for unknown $\phi$ lead to

$$
\begin{aligned}
& \phi=-r_{0} h \frac{x_{2}^{2}}{2}-p_{0} h \frac{x_{1}^{2}}{2}+\frac{n^{2} \lambda^{2} f^{2}}{32 m^{2} A_{11}^{*}} \cos \frac{2 m \pi x_{1}}{a}+\frac{m^{2} f^{2}}{32 n^{2} \lambda^{2} A_{22}^{*}} \cos \frac{2 n \pi x_{2}}{b}- \\
& \frac{\left[B_{21}^{*} m^{4}+\left(B_{11}^{*}+B_{22}^{*}-2 B_{66}^{*}\right) m^{2} n^{2} \lambda^{2}+B_{12}^{*} n^{4} \lambda^{4}-\frac{a^{2}}{\pi^{2}}\left(k_{1} n^{2} \lambda^{2}+k_{2} m^{2}\right)\right]}{A_{11}^{*} m^{4}+\left(A_{66}^{*}-2 A_{12}^{*}\right) m^{2} n^{2} \lambda^{2}+A_{22}^{*} n^{4} \lambda^{4}} \sin \frac{m \pi x_{1}}{a} \sin \frac{n \pi x_{2}}{b} .
\end{aligned}
$$

Substituting the expressions (17) and (18) into Eqs. (13) and using Garlerkin method for the resulting equation yield

$$
\left(D+\frac{B^{2}}{A}\right) f+H f^{2}+K f^{3}-\frac{a^{2} h}{\pi^{2}}\left(r_{0} m^{2}+p_{0} n^{2} \lambda^{2}\right) f+\frac{16 a^{4} h}{m n \pi^{6}}\left(k_{1} r_{0}+k_{2} p_{0}\right)=\frac{16 q_{0} a^{4}}{m n \pi^{6}}
$$

where denote

$$
\begin{aligned}
& A=A_{11}^{*} m^{4}+\left(A_{66}^{*}-2 A_{12}^{*}\right) m^{2} n^{2} \lambda^{2}+A_{22}^{*} n^{4} \lambda^{4}, \\
& B=B_{21}^{*} m^{4}+\left(B_{11}^{*}+B_{22}^{*}-2 B_{66}^{*}\right) m^{2} n^{2} \lambda^{2}+B_{12}^{*} n^{4} \lambda^{4}-\frac{a^{2}}{\pi^{2}}\left(k_{1} n^{2} \lambda^{2}+k_{2} m^{2}\right), \\
& D=D_{11}^{*} m^{4}+\left(D_{12}^{*}+D_{21}^{*}+4 D_{66}^{*}\right) m^{2} n^{2} \lambda^{2}+D_{22}^{*} n^{4} \lambda^{4} \\
& H=\frac{32 m n \lambda^{2}}{3 \pi^{2}} \frac{B}{A}+\frac{8 m n \lambda^{2}}{3 \pi^{2}}\left(\frac{B_{21}^{*}}{A_{11}^{*}}+\frac{B_{12}^{*}}{A_{22}^{*}}\right)-\frac{2 a^{2}}{3 \pi^{4} m n}\left(\frac{k_{2} n^{2} \lambda^{2}}{A_{11}^{*}}+\frac{k_{1} m^{2}}{A_{22}^{*}}\right), \\
& K=\frac{1}{16}\left(\frac{m^{4}}{A_{22}^{*}}+\frac{n^{4} \lambda^{4}}{A_{11}^{*}}\right) ; \lambda=\frac{a}{b} .
\end{aligned}
$$


Eq. (19) derived for odd values of $m, n$ is used to determine buckling loads and postbuckling curves of eccentrically stiffened FGM shallow shells under mechanical loads.

For a shell without stiffeners Eq. (19) reduces to

$$
\begin{aligned}
& {\left[\frac{E_{1} E_{3}-E_{2}^{2}}{E_{1}\left(1-\nu^{2}\right)}\left(m^{2}+n^{2} \lambda^{2}\right)^{2}+\frac{E_{1} a^{4}\left(k_{1} n^{2} \lambda^{2}+k_{2} m^{2}\right)^{2}}{\pi^{4}\left(m^{2}+n^{2} \lambda^{2}\right)^{2}}\right] f-} \\
& -\frac{2 E_{1} a^{2}\left(k_{1} n^{2} \lambda^{2}+k_{2} m^{2}\right)}{3 \pi^{4} m n}\left(1+\frac{16 m^{2} n^{2} \lambda^{2}}{\left(m^{2}+n^{2} \lambda^{2}\right)^{2}}\right) f^{2}+\frac{E_{1}}{16}\left(m^{4}+n^{4} \lambda^{4}\right) f^{3}- \\
& -\frac{a^{2} h}{\pi^{2}}\left(r_{0} m^{2}+p_{0} n^{2} \lambda^{2}\right) f+\frac{16 a^{4} h}{m n \pi^{6}}\left(k_{1} r_{0}+k_{2} p_{0}\right)=\frac{16 a^{4}}{m n \pi^{6}} q_{0} .
\end{aligned}
$$

Introducing

$$
\bar{D}=\frac{D}{h^{3}}, \bar{B}=\frac{B}{h}, \bar{A}=A h, \bar{H}=\frac{H}{h^{2}}, \bar{K}=\frac{K}{h}, \bar{f}=\frac{f}{h} .
$$

the Eqs. (19) can be rewritten by

$$
\begin{aligned}
\left(\bar{D}+\frac{\bar{B}^{2}}{\bar{A}}\right) \bar{f}+\bar{H} \bar{f}^{2}+\bar{K} \bar{f}^{3} & -\frac{1}{\pi^{2}}\left(\frac{a}{h}\right)^{2}\left(r_{0} m^{2}+p_{0} n^{2} \lambda^{2}\right) \bar{f}+ \\
& +\frac{16}{m n \pi^{6}}\left(\frac{a}{h}\right)^{4}\left(h k_{1} r_{0}+h k_{2} p_{0}\right)=\frac{16 q_{0}}{m n \pi^{6}}\left(\frac{a}{h}\right)^{4} .
\end{aligned}
$$

Now investigate the non-linear buckling of reinforced FGM shallow shells in some cases of active loads.

\subsection{Reinforced FGM shallow shell acted on by only lateral pressure $q_{0}$}

A shallow shell considered here may be a cylindrical panel $\left(k_{1}=0, k_{2}=\frac{1}{R}\right)$, a spherical panel $\left(k_{1}=k_{2}=\frac{1}{R}\right)$ or a doubly curved shallow shell $\left(k_{1}=\frac{1}{R_{1}}, k_{2}=\frac{1}{R_{2}}\right)$.

In this case $\left(r_{0}=p_{0}=0\right)$, Eq. (22) representing the load - deflection curve of the shell becomes

$$
q^{*} \equiv \frac{16}{m n \pi^{6}}\left(\frac{a}{h}\right)^{4} q_{0}=\left(\bar{D}+\frac{\bar{B}^{2}}{\bar{A}}\right) \bar{f}+\bar{H} \bar{f}^{2}+\bar{K} \bar{f}^{3} .
$$

The shallow shells only exhibit extremum-type buckling when the material and geometric parameters satisfy specific conditions, i.e. loss of stability occurs at a limit point. The limit points of $q_{0}(\bar{f})$ curves in Eq. (23) are determined by condition

$$
\frac{d q^{*}}{d \bar{f}}=\left(\bar{D}+\frac{\bar{B}^{2}}{\bar{A}}\right)+2 \bar{H} \bar{f}+3 \bar{K} \bar{f}^{2}=0
$$

which yields

$$
\bar{f}_{1,2}=\frac{-\bar{H} \mp\left[\bar{H}^{2}-3 \bar{K}\left(\bar{D}+\frac{\bar{B}^{2}}{\bar{A}}\right)\right]^{1 / 2}}{3 \bar{K}} .
$$


By examining the sign $\frac{d^{2} q^{*}}{d \bar{f}^{2}}$ one obtains the conclusion that the curve represented by Eq. (23) reaches maximum at $\bar{f}_{1}$ with corresponding value

$$
q_{\text {upper }}=\frac{m n \pi^{6}}{16\left(\frac{a}{h}\right)^{4}}\left[\frac{2\left\{\bar{H}^{2}-3 \bar{K}\left(\bar{D}+\frac{\bar{B}^{2}}{\bar{A}}\right)\right\}^{3 / 2}-\bar{H}\left\{9 \bar{K}\left(\bar{D}+\frac{\bar{B}^{2}}{\bar{A}}\right)-2 \bar{H}^{2}\right\}}{27 \bar{K}^{2}}\right]
$$

and reaches minimum value at $\bar{f}_{2}$ with

$$
q_{\text {lower }}=-\frac{m n \pi^{6}}{16\left(\frac{a}{h}\right)^{4}}\left[\frac{2\left\{\bar{H}^{2}-3 \bar{K}\left(\bar{D}+\frac{\bar{B}^{2}}{\bar{A}}\right)\right\}^{3 / 2}+\bar{H}\left\{9 \bar{K}\left(\bar{D}+\frac{\bar{B}^{2}}{\bar{A}}\right)-2 \bar{H}^{2}\right\}}{27 \bar{K}^{2}}\right] \text {. }
$$

The condition providing the existence of the upper and lower buckling loads is

$$
\bar{H}^{2}-3 \bar{K}\left(\bar{D}+\frac{\bar{B}^{2}}{\bar{A}}\right)>0,
$$

i.e. the snap-through behavior of the shallow shell may be predicted and the intensity of the snap-through response is given by the difference between two values (26) and (27). If the material and geometric parameters of the shell are such that the expression (28) becomes an equality, the load-deflection curve has only one stationary point at $\overline{f_{0}}=-\frac{\bar{H}}{3 \bar{K}}$ which can be shown as an inflexion point corresponding to the change of sign of the shell curvature. This change occurs smoothly increasing active load. If the inequation is not satisfied, there exists only one equilibrium shape and load-deflection curve is stable. Note that it needs to put in the expressions $\bar{B}$ and $\bar{H}$ when considering a cylindrical panel $\left(k_{1}=0, k_{2}=\frac{1}{R}\right),\left(k_{1}=k_{2}=\frac{1}{R}\right)$ - for a spherical panel, and $\left(k_{1}=\frac{1}{R_{1}}, k_{2}=\frac{1}{R_{2}}\right)$ - for a doubly curved shallow shell.

\subsection{Reinforced FGM cylindrical panel subjected to an axial compressive load of intensity $r_{0}$}

In this case $\left(p_{0}=q_{0}=0\right), k_{1}=0, k_{2}=\frac{1}{R}$ the equation (21) representing the loaddeflection curve is of the form

$$
\left(\bar{D}+\frac{\bar{B}^{2}}{\bar{A}}\right) \bar{f}+\bar{H} \bar{f}^{2}+\bar{K} \bar{f}^{3}-\frac{m^{2}}{\pi^{2}}\left(\frac{a}{h}\right)^{2} r_{0} \bar{f}=0 .
$$

Because of $\bar{f} \neq 0$, i.e. considering the shell after the loss of stability we obtain

$$
r_{0}^{*} \equiv \frac{m^{2}}{\pi^{2}}\left(\frac{a}{h}\right)^{2} r_{0}=\left(\bar{D}+\frac{\bar{B}^{2}}{\bar{A}}\right)+\bar{H} \bar{f}+\bar{K} \bar{f}^{2} .
$$


Substituting $\bar{f} \rightarrow 0$ in Eq. (30) yields the value of critical load

$$
r_{\text {upper }}=\frac{\pi^{2}}{m^{2}\left(\frac{a}{h}\right)^{2}}\left(\bar{D}+\frac{\bar{B}^{2}}{\bar{A}}\right) .
$$

That is called the upper buckling load, which coincides with the linear buckling load. The lower buckling load of the eccentrically stiffened FGM cylindrical shell can be obtained from equation (30) using the condition

$$
\frac{d r_{0}^{*}}{d f}=\bar{H}+2 \bar{K} \bar{f}=0
$$

which yields $\bar{f}=-\frac{\bar{H}}{2 \bar{K}}$ and the lower buckling load is found as

$$
r_{\text {lower }}=\frac{\pi^{2}}{m^{2}\left(\frac{a}{h}\right)^{2}}\left(\bar{D}+\frac{\bar{B}^{2}}{\bar{A}}-\frac{\bar{H}^{2}}{4 \bar{K}}\right) .
$$

For a cylindrical shell an infinitesimal increase of the upper buckling load (corresponding $\bar{f}=0$ ) leads to rapidly increasing the deflection and decreasing the active load acting on the shell. The deflection suddenly has reached the value $\bar{f} \neq 0$, corresponding to the upper buckling load $r_{\text {upper }}$, at that time the shell curvature has changed, there exists the snap-through phenomenon of the shell. Thus permissible loads have to be chosen such that the safety is provided according to the lower buckling load $r_{l o w e r}$, with such choice the occurrence of collapse in the exploiting process of thin-wall cylindrical panels can be neglected. For an unreinforced FGM cylindrical shell the expressions of buckling loads are obtained from (31) and (32) by omitting factors relating stiffeners.

\subsection{Reinforced FGM plate subjected to lateral compressive loads $r_{0}$ and $p_{0}$}

Repeating all calculations, the upper and lower buckling loads rare of a reinforced FGM plate given similarly (31) and (32)

$$
\begin{aligned}
& r_{\text {upper }}=\frac{\pi^{2}}{\left(m^{2}+\alpha n^{2} \lambda^{2}\right)\left(\frac{a}{h}\right)^{2}}\left(\bar{D}+\frac{\bar{B}^{2}}{\bar{A}}\right), \\
& r_{\text {lower }}=\frac{\pi^{2}}{\left(m^{2}+\alpha n^{2} \lambda^{2}\right)\left(\frac{a}{h}\right)^{2}}\left(\bar{D}+\frac{\bar{B}^{2}}{\bar{A}}-\frac{\bar{H}^{2}}{4 \bar{K}}\right) .
\end{aligned}
$$

where $\alpha=\frac{p_{0}}{r_{0}}$, but need to put $k_{1}=k_{2}=0$ in the expressions $\bar{B}$ and $\bar{H}$.

For a FGM plate without stiffeners $\bar{B}=0, \bar{H}=0$ there exists only the buckling load

$$
r_{c r}=\frac{\pi^{2}}{a^{2} h} \frac{E_{1} E_{3}-E_{2}^{2}}{E_{1}\left(1-\nu^{2}\right)} \frac{\left(m^{2}+n^{2} \lambda^{2}\right)^{2}}{\left(m^{2}+\alpha n^{2} \lambda^{2}\right)} .
$$

That means, for a FGM plate post-buckling behaviors of reinforced and unreinforced plates are different. For an unreinforced FGM plate, gradually increasing the active load 
leads to increasing the deflection, there isn't an unstable domain, while for a reinforced plate there exists yet an unstable domain.

\section{NUMERICAL EXAMPLES}

To validate the present formulation in buckling and post-buckling of reinforced FGM shallow shells under mechanical loads, the nonlinear response of a simply supported FGM cylindrical panel without stiffeners under uniform external pressure is analyzed, which was considered by Zhao and Liew [19] using the element-free kp-Ritz method and modified version of Sander's nonlinear shell theory.

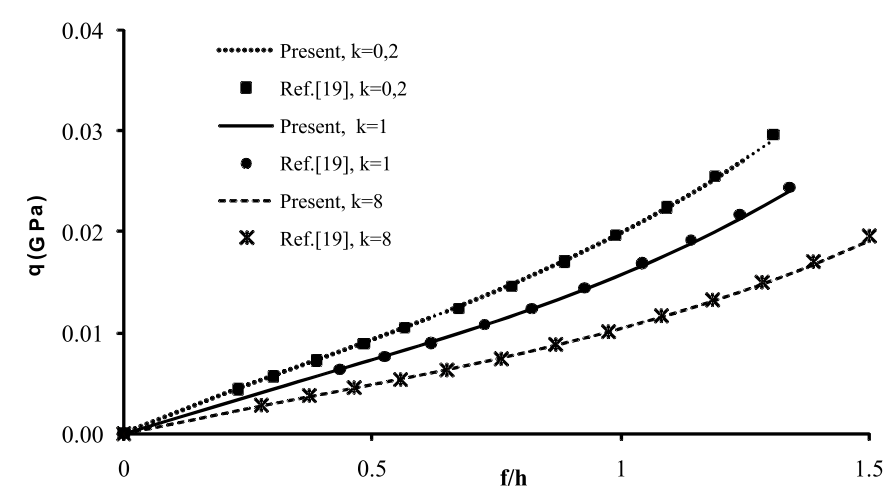

Fig. 2. Comparison of non-linear load-deflection curves for unreinforced FGM cylindrical panels with theoretical results reported by Zhao and Liew [19]

The non-linear load-deflection curves of unreinforced panels made of Zirconia $\left(\mathrm{ZrO}_{2}\right)$ and Aluminum $(\mathrm{Al})$ with different values of volume fraction index $k$ are compared in Fig. 2 with Zhao and Liew's results. As can be observed, a very good agreement is obtained in this comparison study.

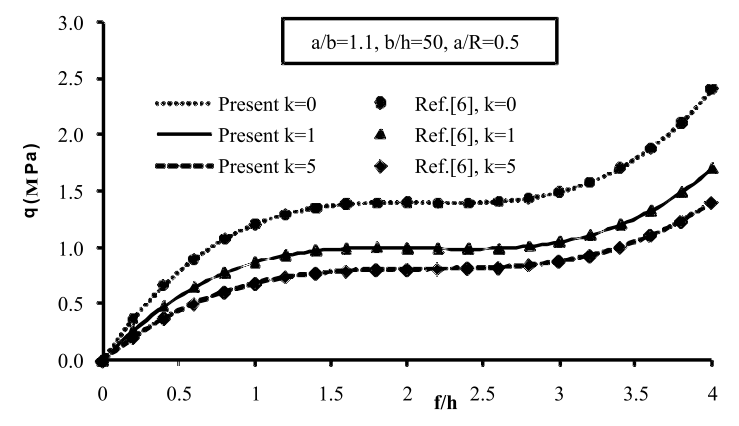

Fig. 3. Comparison of non-linear load-deflection curves for unreinforced FGM cylindrical panels with theoretical results reported by Duc and Tung [6]

As a second comparison study, postbuckling of a simply supported unreinforced $\mathrm{ZrO} 2 / \mathrm{Al}$ cylindrical panel under uniform lateral pressure and with three different values 
of volume fraction index $k=(0,1$ and 5$)$ is considered, that was also analyzed by Duc and Tung [6]. It is evident that good agreement is achieved (see Fig. 3)

Now to illustrate the proposed approach to eccentrically stiffened FGM plates and shallow shells, we consider these structures made of FGM that consist of Zirconia and aluminum with the following properties [19]: $E_{c}=151 \mathrm{GPa}$ and $E_{m}=70 \mathrm{GPa}$ and Poisson's ratio is chosen to be 0.3 .

Effects of material parameters as well as stiffeners on the critical buckling loads and post-buckling curves of reinforced and unreinforced FGM plates, cylindrical panels and spherical panels are shown in Tables $1-4$ and Figs. 4 - 9. It is noted that in all figures $f / h$ denotes the dimensionless maximum deflection of the shell.

Table 1. Critical buckling loads of FGM plates under uniaxial comporessive load

\begin{tabular}{|c|c|c|c|c|}
\hline$r_{0}^{c r}(\mathrm{~Pa})$ & \multicolumn{2}{|c|}{ Unreinforced } & \multicolumn{2}{c|}{ Reinforced } \\
\cline { 2 - 5 }$k$ & Upper & Lower & Upper & Lower \\
\hline 0 & 13647529.90 & 13647529.90 & 14547913.82 & 14545345.33 \\
\hline 1 & 9539897.81 & 9539897.81 & 10681832.44 & 10678151.02 \\
\hline 5 & 8116402.01 & 8116402.01 & 9232825.32 & 9228001.74 \\
\hline$\infty$ & 6326669.49 & 6326669.49 & 7198149.50 & 7192710.31 \\
\hline
\end{tabular}

Table 1 gives values of critical buckling loads of unreinforced and reinforced plates respectively under uniaxial compressive load with four different values of volume fraction index $k=(0,1,5$ and $\infty)$ geometric parameters of plate and stiffeners considered here are: $\lambda=1, a=2, h=0.01$, spacings, width and eccentricities of stiffeners $s_{1}=s_{2}=0.4, \delta=$ 0.003 and $z_{1}=z_{2}=0.0225(\mathrm{~m})$, respectively.

The critical buckling loads i.e the minimum buckling loads of FGM plates corresponds to $m=n=1$ that is the first buckling shape mode. As can be seen they decrease as the $k$ increases as expected, and critical buckling loads of reinforced plates are greater than those of unreinforced plates. For unreinforced plates there exists only one value of critical load, but for reinforced plates occur upper and lower critical loads as shown in Eq. (33) and Table 1.
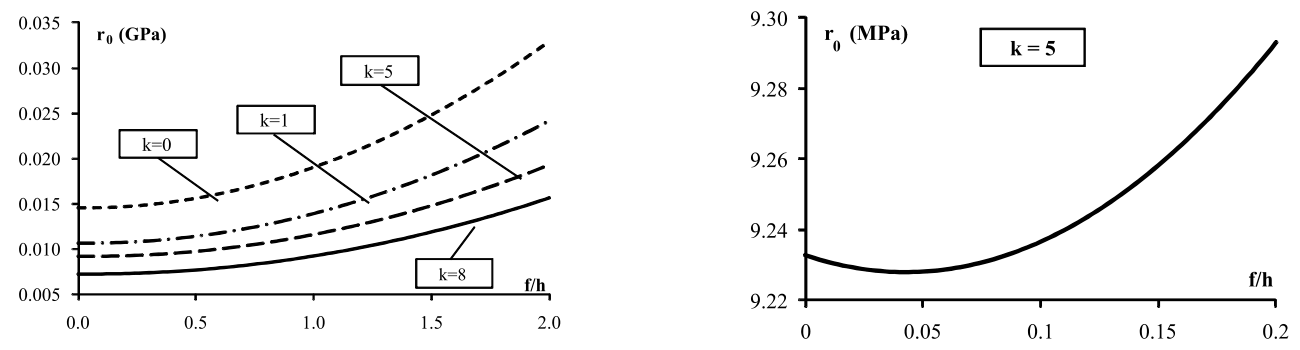

Fig. 4. Postbuckling curves of reinforced FGM plates under uniaxial compressive load vs $k$

Fig. 4 shows postbuckling load-deflection curves of reinforced FGM plate under uniaxial compressive load versus four different values of volume fraction index $k=(0,1,5$ and 
$\infty)$, (where $k=\infty$ corresponding to full metal plate). As can be observed the postbuckling curves become lower as the $k$ increases.

Comparison of postbuckling curves of reinforced and unreinforced FGM plates with the value $k=5$ is given in Fig. 5 .

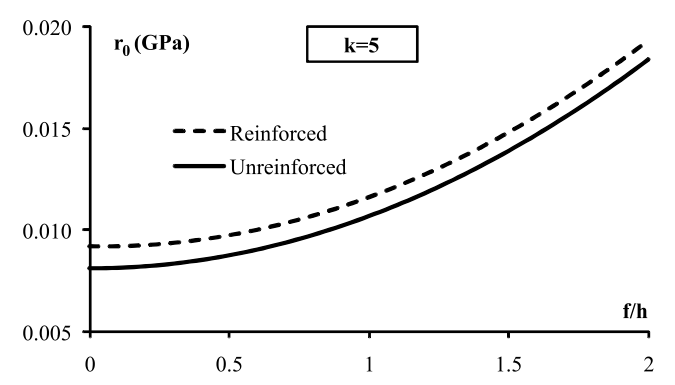

Fig. 5. Comparison of postbuckling curves of reinforced and unreinforced FGM plates under uniaxial compressive load

Postbuckling load-carrying capability of the reinforced plate is higher than that of unreinforced plate.

Table 2. Critical buckling loads of FGM cylindrical panels under axial compressive load

\begin{tabular}{|c|c|c|c|c|}
\hline$r_{0}^{c r}(\mathrm{~Pa})$ & \multicolumn{2}{|c|}{ Unreinforced } & \multicolumn{2}{c|}{ Reinforced } \\
\cline { 2 - 5 }$k$ & Upper & Lower & Upper & Lower \\
\hline 0 & $9.299 \mathrm{E}+07$ & $9.266 \mathrm{E}+07$ & $9.697 \mathrm{E}+07$ & $9.665 \mathrm{E}+07$ \\
\hline 1 & $6.681 \mathrm{E}+07$ & $6.657 \mathrm{E}+07$ & $7.183 \mathrm{E}+07$ & $7.160 \mathrm{E}+07$ \\
\hline 5 & $5.300 \mathrm{E}+07$ & $5.282 \mathrm{E}+07$ & $5.787 \mathrm{E}+07$ & $5.770 \mathrm{E}+07$ \\
\hline$\infty$ & $4.311 \mathrm{E}+07$ & $4.296 \mathrm{E}+07$ & $4.69 \mathrm{E}+07$ & $4.67 \mathrm{E}+07$ \\
\hline
\end{tabular}

The upper and lower critical buckling loads of reinforced and unreinforced FGM cylindrical panels with given geometrical parameters: $\lambda=1, a=2, h=0.01, R=10, s_{1}=$ $s_{2}=0.4, \delta=0.003, z_{1}=z_{2}=0.0225(\mathrm{~m})$ and various values $k$ under axial compressive load are achieved with buckling mode $m=3, n=1$ and given in the Table 2 .

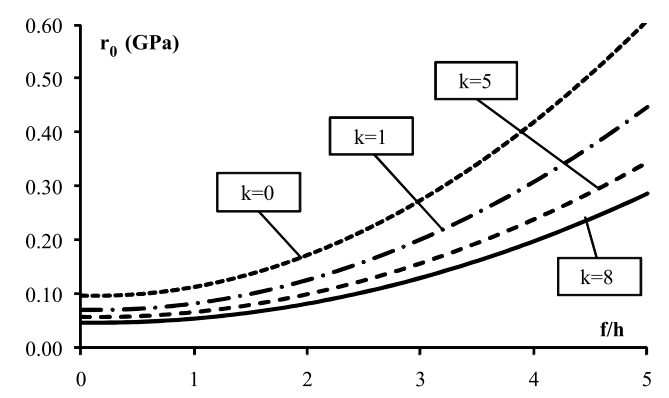

Fig. 6. Effect of volume fraction index on the nonlinear response of reinforced FGM cylindrical panels under axial load 
Postbuckling curves of reinforced FGM cylindrical panel with various values $k$ are shown in Fig. 6. Clearly can be seen that stiffeners enhance the stability and the postbuckling load-carrying capability of cylindrical panels.

Table 3. Critical buckling loads of FGM cylindrical panels under pressure load

\begin{tabular}{|c|c|c|c|c|}
\hline$q_{0}^{c r}(P a)$ & \multicolumn{2}{|c|}{ Unreinforced } & \multicolumn{2}{c|}{ Reinforced } \\
\cline { 2 - 5 }$k$ & Upper & Lower & Upper & Lower \\
\hline 0 & $2.597 \mathrm{E}+04$ & $2.130 \mathrm{E}+04$ & $2.728 \mathrm{E}+04$ & $2.374 \mathrm{E}+04$ \\
\hline 1 & $1.884 \mathrm{E}+04$ & $1.525 \mathrm{E}+04$ & $2.027 \mathrm{E}+04$ & $1.790 \mathrm{E}+04$ \\
\hline 5 & $1.458 \mathrm{E}+04$ & $1.219 \mathrm{E}+04$ & $1.603 \mathrm{E}+04$ & $1.476 \mathrm{E}+04$ \\
\hline$\infty$ & $1.204 \mathrm{E}+04$ & $9.872 \mathrm{E}+03$ & $1.336 \mathrm{E}+04$ & $1.224 \mathrm{E}+04$ \\
\hline
\end{tabular}

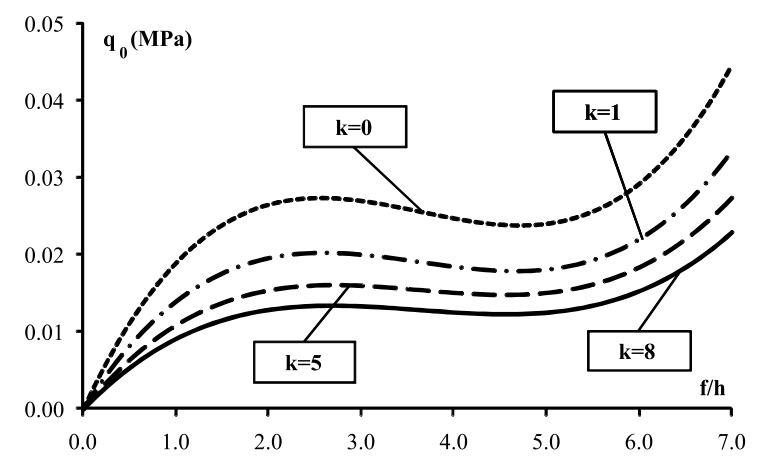

Fig. 7. Effect of volume fraction index on the nonlinear response of reinforced FGM cylindrical panels under pressure load

The critical buckling loads and postbuckling load - deflection curves of reinforced and unreinforced FGM cylindrical panels with the same geometrical parameters and various values of volume fraction index $k$ under pressure load are given in the Table. 3 and Fig. 7 , respectively.

As expected, a benign snap-through behavior of the panels is shown in this figure, the postbuckling curves become higher for smaller values of $k$ representing panels with the greater volume percentage of zirconia. The critical buckling loads of reinforced panels are greater than those of unreinforced ones. It is evident from two Table 2 and Table 3 that loading carrying capacity of the cylindrical panel under axial compressive load is better than that of panel under pressure load.

Fig. 8 gives nonlinear postbuckling curves of reinforced spherical panel with geometrical parameters $\lambda=1, a=2, h=0.01, R_{1}=R_{2}=10, s_{1}=s_{2}=0.4, \delta=0.003, z_{1}=z_{2}=$ $0.0225(\mathrm{~m})$ and various values $k$ under pressure load, that leads to the similar conclusion such as for cylindrical panel.

The comparison of critical loads and postbuckling curves of reinforced FGM cylindrical panel and spherical panel with the same material and geometrical parameters under 


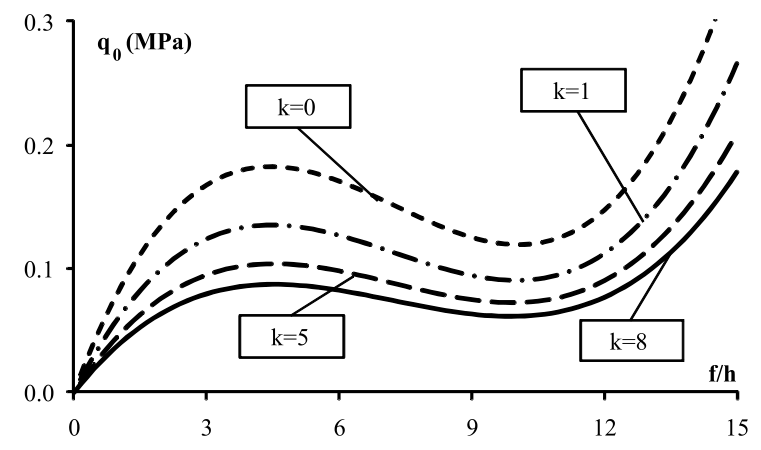

Fig. 8. Effect of volume fraction index on the nonlinear response of reinforced FGM spherical panels under pressure load

Table 4. Comparison of critical buckling loads of cylindrical panel and spherical panel subjected to pressure load

\begin{tabular}{|c|c|c|c|c|}
\hline \multirow{2}{*}{$\begin{array}{c}q_{0}^{c r}(\mathrm{~Pa}) \\
k\end{array}$} & \multicolumn{2}{|c|}{ Cylindrical panel } & \multicolumn{2}{c|}{ Spherical panel } \\
\cline { 2 - 5 } & Upper & Lower & Upper & Lower \\
\hline 0 & $2.597 \mathrm{E}+04$ & $2.130 \mathrm{E}+04$ & $1.788 \mathrm{E}+05$ & $1.084 \mathrm{E}+05$ \\
\hline 1 & $2.027 \mathrm{E}+04$ & $1.790 \mathrm{E}+04$ & $1.351 \mathrm{E}+05$ & $9.046 \mathrm{E}+04$ \\
\hline 5 & $1.458 \mathrm{E}+04$ & $1.219 \mathrm{E}+04$ & $9.926 \mathrm{E}+04$ & $6.080 \mathrm{E}+04$ \\
\hline$\infty$ & $1.336 \mathrm{E}+04$ & $1.224 \mathrm{E}+04$ & $8.72 \mathrm{E}+04$ & $6.14 \mathrm{E}+04$ \\
\hline
\end{tabular}

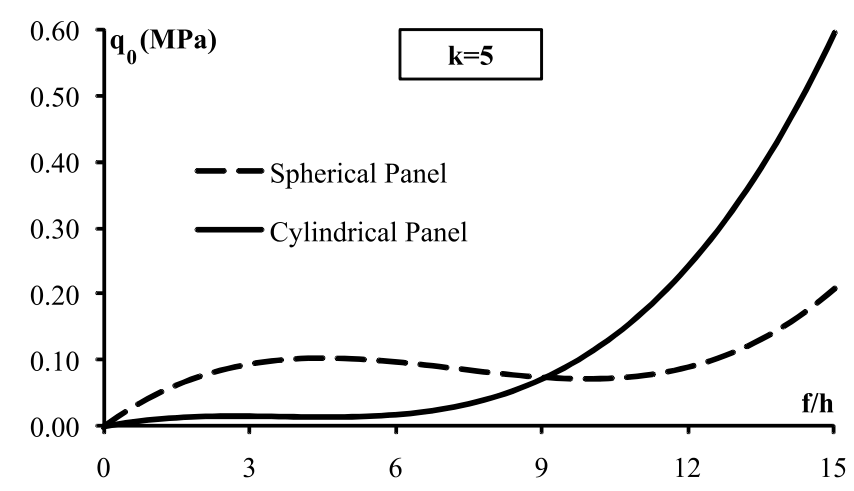

Fig. 9. Comparison of postbuckling curves of reinforced cylindrical and spherical panels under pressure load with $k=5$

pressure load (see Table 4 and Fig. 9) leads to a conclusion on the advantage of pressureloaded spherical panels. 


\section{CONCLUSIONS}

A formulation of the governing equations of eccentrically reinforced functionally graded plates and shallow shells based upon the classical shell theory and the smeared stiffeners technique with von Karman-Donnell nonlinear terms has been presented.

By use of Galerkin method, explicit expressions of critical buckling loads and nonlinear load-deflection curves for simply supported eccentrically stiffened FGM plates and shallow shells under various loadings are determined. The nonlinear responses of plates and panels are complex and significantly influenced by material parameters. Stiffeners enhance the stability and load-carrying capacity of plates and shells.

Reinforced spherical panel works better than the cylindrical one under pressure load; the load-carrying capacity of cylindrical panel under axially compressive load is greater than that under pressure load.

\section{REFERENCES}

[1] Birman V., Buckling of FGM hybrid composite plates, Proc. of $10^{\text {th }}$ Conf. on Eng. Mech, USA, (1995).

[2] Feldman E., Aboudi J., Buckling analysis of FGM plates subjected to uniaxial loading, Composite Structures, 38 (1997), 29 - 36.

[3] Tung H. V., Duc N. D., Nonlinear analysis of stability for FGM plates under mechanical and thermal loads, Composite Structures, 92 (2010) 1184 - 1191.

[4] Shen H. S., Postbuckling analysis of axially loaded FGM cylindrical panels in thermal environments, Int. J. Solids Structures, 39 (2002), 5991 - 6010.

[5] Shen H. S., Leung A. Y. T., Postbuckling of pressure - loaded FGM cylindrical panels in thermal environments, J. Eng. Mech. ASCE, 129(4) (2003), $414-425$.

[6] Duc N. D., Tung H. V., Nonlinear response of pressure - loaded FGM cylindrical panels with temperature effects, Composite Structures, 92 (2010), 1664 - 1672.

[7] Yang Y., Liew K. M., Wu Y. F., Kitipornchai S., Thermo - mechanical postbuckling of FGM cylindrical panels with temperature dependent properties, Int. J. Solids Structures, 43 (2006), $307-324$.

[8] Li S. I., Batra R. C., Buckling of axially compressed thin cylindrical shells with FGM middle layer, Thin Wall Structures, 44 (2006), 1039 - 1047.

[9] Shen H. S., Postbuckling analysis of pressure - loaded FGM cylindrical shells in thermal environments, Engineering Structures, 25 (2003), 487 - 497.

[10] Huang H., Han Q., Research on nonlinear postbuckling of FGM cylindrical shells under radial loads, Composite Structures, 92 (2010), 1352 - 1357.

[11] Bich D. H., Nonlinear buckling analysis of FGM shallow spherical shells, Vietnam Journal of Mechanics, 31 (2009), 17 - 30.

[12] Naj R., Sabzikar M., Eslami M. R., Thermal and mechanical instability of FGM truncated conical shells, Thin Wall Structures, 46 (2008), $65-78$.

[13] Sofiyev A. H., The stability of FGM ceramic - metal cylindrical shells under aperiodic axial impulse loading, Composite Structures, 69 (2005), 247 - 257.

[14] Ng T. Y., Lam K. Y., et al. Dynamic stability analysis of FGM cylindrical shells under periodic axial loading, Int. J. Solids Structures, 38 (2001), 1295 - 1309.

[15] Darabi M., et al, A nonlinear analysis of dynamic stability for FGM cylindrical shells under periodic axial loading, Composite Structures, 82 (2008), 201 - 211. 
[16] Bich D. H., Long V. D., Nonlinear dynamical analysis of imperfect FGM shallow shells, Vietnam Journal of Mechanics, 32 (2010), 1 - 14.

[17] Ganapathi M., Dynamic stability characteristics of FGM shallow spherical shells, Composite Structures, 79 (2007), 338 - 343.

[18] Brush D. D., Almroth B. O., Buckling of bars, plates and shells, McGraw - Hill (1975).

[19] Zhao X., Liew K. M., Geometrically nonlinear analysis of FGM shells, Int. J. Mech. Sci., 51 (2009), $131-144$. 